\title{
Promises and pitfalls of cannabinoids as agents with potential anticancer efficacy
}

\author{
Lara A. Haeusser, Lothar Kanz, Marcus M. Schittenhelm, Kerstin M. Kampa-Schittenhelm*
}

University Hospital Tübingen, Dept. of Oncology, Hematology, Clinical Immunology, Rheumatology and Pulmology, Tübingen, Germany

Article Info

\section{Article Notes}

Received: August 12, 2016

Accepted: September 09, 2016

\section{*Correspondence:}

Dr. Kerstin M. Kampa-Schittenhelm, Medizinisches Universitätsklinikum Tübingen, Dept. of Oncology, Hematology, Clinical Immunology, Rheumatology and Pulmology, Laboratory of experimental Hematology/Oncology, Otfried-Müller-Straße 10, BB West, Rooms 585-587, 72076 Tübingen, Germany, Telephone: (+49) 7071298 0676; Fax: (+49) 7071 294932, Email: kerstin.kampa-schittenhelm@med.uni-tuebingen.de

C 2016 Kampa-Schittenhelm KM. This article is distributed under the terms of the Creative Commons Attribution 4.0 International License

\section{Keywords:}

Delta9-Tetrahydrocannabinol

Dronabinol

THC

Cannabidiol

CBD

\section{ABSTRACT}

The endocannabinoid system is extensively studied in neuroscience and clinical use of cannabinoid derivatives as substances with remarkable spasmolytic effects in multiple sclerosis and antiemetic potential in cancer therapy as well as pain-relieving properties is broadly acknowledged.

However, it becomes increasingly apparent, that in addition cannabinoids exert manifold functions in various organ systems, such as the immune system, the reproductive or cardiovascular system among others. Moreover, interactions with signaling pathways involved in programmed cell death, angiogenesis, metastasis or anti-tumor immunity make it highly suggestive that cannabinoids may have therapeutic potential in the treatment of cancer. Indeed, detailed reports have repeatedly shown anticancer efficacy in solid and hematologic tumor models, best characterized in human gliomas.

Anecdotal evidence of blast control in a young patient with acute myeloid leukemia has led us to systematically investigate the potential use of cannabinoids in the treatment of acute leukemia.

These data are summarized herein in the context of key data regarding anticancer efficacy of cannabinoids.

\section{Background}

Even though the use of cannabinoids as medicinal products has an almost 5000 year old documented tradition dating back to the Chinese emperor Shen Nung ${ }^{1}$, and clinical efficacy with respect to spasmolytic activity and pain relief, is recognized since the early $19^{\text {th }}$ century even in European academic medicine - today clinical use is still under intense debate and research is hampered by legal restrictions in many countries.

Meanwhile more than 100 natural cannabinoid derivatives are $\mathrm{known}^{2,3}$. Of these, especially Delta9-Tetrahydrocannabinol (THC), the major psychoactive constituent of Cannabis sativa, and Cannabidiol (CBD), a non-psychoactive derivative, are currently in the focus of research.

Positive effects of cannabinoids have been suggested for many diseases including, but not limited to neurologic, autoimmune, inflammatory and mental disorders ${ }^{4}$.

Several medicinal products containing cannabinoids have gained clinical approval by the authorities, such as Marinol (THC) in the U.S. for the treatment of chemotherapy-induced nausea, vomiting and cachexia in AIDS patients or Sativex (THC/CBD) in Germany for spasmolysis in multiple sclerosis.

Additionally, there is recurring evidence that cannabinoids possess tumor-regressive effects, including leukemia - dating back to scientific reports in the $1970 \mathrm{~s}^{5,6}$. Since that time various 
cannabinoid derivatives have been evaluated in numerous tumor models. However, interpretation of the data is challenging: Conflicting opinions exist as to whether the endocannabinoid system exerts a tumor-suppressor or rather an oncogenic role $\mathrm{e}^{3,7,8}$ and references within.

Taking a closer look at the available data, it becomes apparent that these discrepancies are, at least in part, due to the diversity of cell and animal models studied, the pharmacological differences in receptor binding, specificity or mode of experimental applications of the respective cannabinoid derivative tested, as well as the different tumor entities investigated, which does not allow to draw general conclusions.

Nevertheless, the overwhelming majority of reports suggest that cannabinoids in fact exhibit anti-tumorous properties via inhibition of proliferation, induction of autophagy and/or apoptosis, immunomodulatory effects and inhibition of angiogenesis, cell invasion as well as metastasis in in vitro and in vivo models ${ }^{5,7-14}$.

\section{Mode-of-action}

Cannabinoids signal trough G-protein-coupled cannabinoid receptors (CB). Physiologically, $\mathrm{CB}_{1}$ receptors are predominantly localized in brain tissues whereas $\mathrm{CB}_{2}$ receptors are preferably found in the lymphatic system, brain, brain endothelium, bone and skin ${ }^{11,15-22}$.

In addition, $\mathrm{CB}_{1 / 2}$ receptor-independent signaling has been discussed for several cannabinoid derivatives: THC, CBD as well as endogenous anandamide have been demonstrated to additionally signal via the orphan $G$ protein-coupled receptor 55 (GPR55). Notably, the capsaicin receptor TRPV1 (Transient Receptor Potential Cation Channel Subfamily V Member 1, aka Vanilloid Receptor 1 ), frequently co-localizing with $\mathrm{CB}_{1}$ or $\mathrm{CB}_{2}$, is also a target of CBD and anandamide $8,18,23-26$. However, distribution and function of these and potentially other additional cannabinoid receptors are still under investigation.

Interestingly, while CB receptors physiologically display distinct tissue-specific distribution patterns, high expression levels of $\mathrm{CB}_{1}$ as well as $\mathrm{CB}_{2}$ receptors are frequently found in cancer - irrespective of distribution levels in the corresponding physiologic tissue $e^{9,19,20,27}$.

The significance of dysregulation of $\mathrm{CB}$ receptor expression in tumor tissue - or the endocannabinoid system in its entirety, including endogenous ligands and endocannabinoid-metabolizing enzymes, is still vague $^{3,7,8,27,28}$. Some work suggest a direct contribution to tumorigenesis and tumor aggressiveness ${ }^{28-32}$. In contrast, competing data describe an anti-tumorigenic rescue mechanism of the endocannabinoid system in an attempt to induce programmed cell death and antiproliferation in cancer cells ${ }^{8-10,30,33,34}$.
To make it even more complex - accumulating evidence suggests, that in cells that physiologically express CB receptors at similar densities as cancer cells, distinct different pathways are activated upon cannabinoid exposition resulting in different cell fate, specifically induction of apoptosis in tumor tissue - whereas physiologic tissue remains unaffected by apoptosis ap, $^{3,11,12,35-39}$.

Most knowledge regarding the anti-tumor activity of cannabinoids and their signaling pathways stems from glioblastoma research: Here it was demonstrated that cannabinoids induce apoptosis in glioma cells via stimulation of de novo synthesis of pro-apoptotic sphingolipid ceramide in a $\mathrm{CB}_{1}-$ and $\mathrm{CB}_{2}$-dependent manner ${ }^{11,40,41}$. Downstream, THC treatment leads to inhibition of the mechanistic target of rapamycin complex 1 (mTORC1), finally resulting in autophagy-induced celldeath. This complex multistep process includes inhibition of AKT signaling via upregulation of the transcriptional regulator, stress-regulated protein p8 (NUPR1), which has been postulated to be involved in the control of tumorigenesis and tumor progression ${ }^{3,35,36,42-45}$.

Distinct signaling pathways of other tumor entities upon cannabinoid exposure are currently under investigation. For example, in colorectal cancer cells inhibition of RASMAPK as well as PI3K/AKT survival signaling pathways and in acute leukemia involvement of the MAPK/ERK pathways was demonstrated ${ }^{46-49}$.

As a consequence, multiple cellular functions influencing cellular viability and integrity are affected, leading to cell cycle arrest, inhibition of proliferation, induction of autophagy or apoptosis and impairment of cellular migration $3,8,11,35,36,40-43,50$ and references within.

For acute lymphoblastic as well as myeloid leukemia our group has recently shown, that subcohorts of acute leukemia highly express $\mathrm{CB}_{1}$ and $\mathrm{CB}_{2}$ receptors. Intriguingly, we were able to demonstrate that $\mathrm{CB}_{1 / 2}$ expression of a broad range of leukemia cell line models as well as freshly isolated patient derived blasts was a prerequisite for potent pro-apoptotic response to THC treatment. Notably, healthy donor mononuclear cells and patient blasts not responding to THC displayed significantly lower $\mathrm{CB}_{1 / 2}$ expression levels. Using selective $\mathrm{CB}_{1 / 2}$-receptor antagonists as well as a CRISPR double nickase knockdown approach, we confirmed receptor dependent induction of apoptosis in the $\mathrm{CB}_{1 / 2}$ expressing cell lines and patient samples ${ }^{12}$.

Additionally, subcohort analysis revealed that antileukemic activity was preferentially observed in blasts with lymphoid differentiation or myeloid blasts aberrantly expressing lymphatic antigens ${ }^{12}$. 
Since it has been speculated, that anti-leukemogenic doses of THC might not be achieved in vivo this topic was addressed by undertaking a plasma inhibitory test: A reference cell line with well-established sensitivity towards THC (Jurkat) was cultured in serum extracted from a patient treated with dronabinol (THC) 2x6 drops/ day of a 2,5\% oily solution for best supportive care reasons (emesis and tumor cachexia). At 48 hours post exposition to the patient's serum, an IC50 was reached for the Jurkat cell line, confirming that anti-leukemic efficacy is indeed achievable in vivo ${ }^{12}$.

On a side note, and together with the shown antileukemic, antiemetic and appetite stimulant efficacies attributed to cannabinoids, another promising interesting field of application might be the clinical evaluation of cannabinoids in the setting of allogeneic transplantation concepts. Due to its anti-inflammatory properties first data suggest a positive effect with respect to graft versus host disease (GVHD): A mouse model for intestinal GVHD revealed intriguing responses towards treatment with $\mathrm{THC}^{51}$. Even more, a recent phase II trial using CBD in combination with standard prophylaxis resulted in decreased incidence of acute GVHD ${ }^{52}$.

\section{Clinical experience}

Despite the fact, that cannabinoids are being intensively investigated in cancer research for over 40 years, resulting in a large body of evidence for the anti-tumorigenic potential of some cannabinoid derivatives, to our knowledge, so far there has been only one clinical trial evaluating the safety and antitumor efficacy of cannabinoids in human malignancies in vivo: A pilot phase I study was conducted using THC in patients with advanced, recurrent glioblastoma multiforme. Though not statistically significant (due to the small number of patients treated) some partial responses were noted in 9 evaluable patients. Importantly, the above described downstream signal transduction pathways identified in previous in vitro studies were successfully confirmed in patient tumor tissue in vivo ${ }^{27}$.

In acute leukemia, there is evidence of clinical efficacy coming from a case report of a 14-year-old girl suffering from a highly aggressive, refractory form of acute lymphoblastic leukemia $(B C R-A B L 1 \text { positive, Philadelphia-ALL })^{53}$. This patient was treated with Hemp oil extracts obtained from outside sources for best supportive care. Intriguingly, a rapid, dose-dependent decrease of leukocytes (starting with WBC $>300000 /$ microL) until blast clearance in the peripheral blood was observed. Importantly, cannabinoid therapy was well tolerated ${ }^{53}$.

Depending on the particular hemp oil strain administered, antileukemic efficacies varied - bringing back the focus to the diverging antitumor efficacies of cannabinoid derivatives described in preclinical models.
Again, this notion states the importance of rigorously comparing and testing standardized therapeutics in defined tumor models.

It is utterly remarkable, that the selected case fits into the defined responder cohort of our own study. Thus, these data support the systematic evaluation of THC in a clinical trial for advanced acute leukemia - which is currently planned at our institution.

Together with the abundance of preclinical data, the available clinical data support the systematic evaluation of other tumor entities as well. Currently, two promising clinical trials are evaluating CBD in solid tumors and the combination of THC/CBD (Sativex®) with temozolomide in glioblastoma (https://clinicaltrials.gov, NCT02255292, NCT01812603 Part A/NCT01812616 Part B/placebo).

\section{Outlook}

Cannabinoids bear potential clinically relevant antitumor efficacy. However, it becomes increasingly clear, that choice of cannabinoid derivative and tumor entity may significantly alter therapy outcome - and may even deteriorate course of disease when administered in the wrong context or using insufficient doses.

Systematic preclinical and clinical evaluation is mandatory to definitely define patient cohorts benefitting from such therapies.

Preconceptions and legal restrictions due to the unwanted psychoactive efficacy of some cannabinoids have hampered basic research and clinical evaluation for decades.

Of note, as we and others have reported before, unwanted psychotropic effects can be minimized by slowly building up the patient's tolerance towards cannabinoids by a dropwise titration of administered doses. On the other hand, it should not remain unmentioned that possible sideeffects like stimulation of appetite, as well as relief of pain, anxiety and stress may as well profoundly contribute to the well-being of a terminally ill tumor patient.

Alternatively, non-psychoactive ( $\mathrm{CB}_{1}$-independent) components like CBD may be of special interest. Again, systematic evaluation of CBD-specific mode-of-action is needed, which may diverge from other cannabinoids. To date, controversial opinions exist as to whether CBD acts receptor-independent, as a receptor agonist on $\mathrm{CB}_{2}$ and/or signals via alternative receptors ${ }^{3,54,55}$.

Regarding the potential antileukemic efficacy, our preliminary data suggest an at least equipotent to superior effect of CBD compared to THC with respect to induction of cell death. FIGURE 1 shows an annexin V-based apoptosis assay comparing CBD and THC in two leukemia cell models, the T-lymphoblastic leukemia Jurkat cell line and the acute myelogenous leukemia cell line MOLM13. 


\section{Proapoptotic efficacy of THC and CBD in leukemia cells}

\section{A}

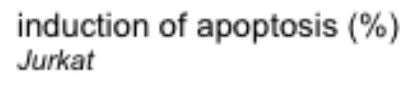

-THC $\square \mathrm{CBD}$

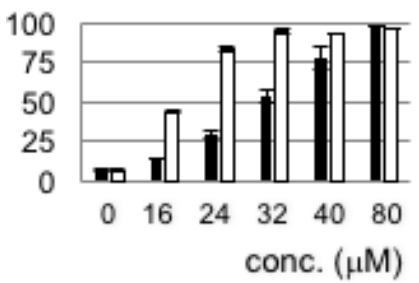

B

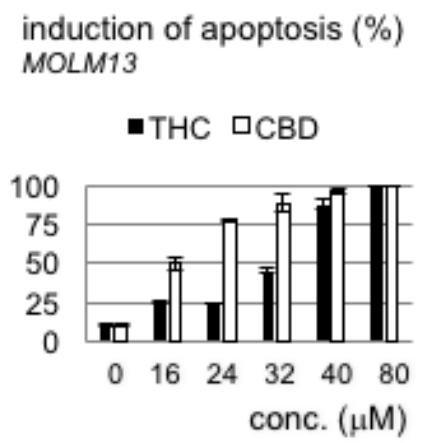

Figure 1: Proapoptotic efficacy of THC and CDB in leukemia cells.

Comparison of proapoptotic effects of THC and CBD in two acute leukemia cell lines using an annexin V/propidium iodide-based flow cytometry approach shows potent efficacy of both cannabinoids - with a benefit of CBD in this model. Cells were treated with THC or $\mathrm{CBD}$ in equivalent concentrations for 48 hours. Drug-carrier control assays, which did not reveal any significant antileukemic effects at the highest concentrations were provided earlier ${ }^{12}$.

Further, a combination approach of cannabinoids and standard (chemo)therapeutics may be attractive as suggested by several groups. THC or synthetic cannabinoids (like GW, ACPA, SR1) were beneficially combined with temozolomide in glioma ${ }^{56}$ or gemcitabine in pancreatic cancer cell models ${ }^{57}$, including resensitization of otherwise chemotherapy-resistant cells ${ }^{56}$.

For acute lymphoblastic leukemia models a beneficial synergistic antileukemic effect was suggested for the combination of THC with cytarabine, anthracyclines and vincristine ${ }^{46}$. However, own preliminary data using leukemia cell models, do not support universal value of combination approaches. This may be due to unforeseen interactions of different substances and cannabinoids (unpublished data). Detailed preclinical research is highly recommended to define optimal combination strategies.

To summarize, there is mounting evidence that cannabinoids as low-toxic agents may have attractive anticancer/antileukemic efficacy and may be especially of interest in the context of heavily pre-treated and therapy refractory, elderly, comorbid or terminally ill patients.

Systematic definition of optimal cannabinoid derivatives, combination strategies and tumor entities benefiting from such an approach need to be the focus of future research including consequent evaluation in clinical trials to fill the gap of 5000 years of empiric medicinal cannabis use.

\section{Acknowledgments}

Grant support in part by the Brigitte Schlieben-Lange Programm des Ministeriums für Wissenschaft, Forschung und Kunst des Landes Baden-Württemberg (KKS) and the Athene Programm der Exzellenzinitiative der Universität Tübingen (KKS). We acknowledge support by the Deutsche
Forschungsgemeinschaft and Open Access Publishing Fund of the University of Tübingen.

Due to space limitations we were not able to reference and/or discuss all relevant publications in detail and concentrated on providing an overview - focusing on acute leukemia.

\section{References}

1. Emboden WA. Cannabis in Ostasien - Herkunft, Wanderung und Gebrauch. In: Völger G, (ed.). Rausch und Realität, Drogen in den frühen Hochkulturen. Reinbek bei Hamburg: Rowohlt, 1982, p. 557-66.

2. Pertwee RG. Emerging strategies for exploiting cannabinoid receptor agonists as medicines. British Journal of Pharmacology. 2009; 156: 397-411.

3. Velasco G, Sánchez C, Guzmán M. Anticancer mechanisms of cannabinoids. Current Oncology. 2016; 23: S23-S32.

4. Hirst RA, Lambert DG, Notcutt WG. Pharmacology and potential therapeutic uses of cannabis. British journal of anaesthesia. 1998; 81: 77-84.

5. Munson A, Harris L, Friedman M, Dewey W, Carchman R. Antineoplastic activity of cannabinoids. Journal of the National Cancer Institute. 1975.

6. Tucker AN, Friedman MA. Effects of cannabinoids on L1210 murine leukemia. 1. Inhibition of DNA synthesis. Research communications in chemical pathology and pharmacology. 1977; 17: 703-14.

7. Velasco G, Sánchez C, Guzmán M. Towards the use of cannabinoids as antitumour agents. Nat Rev Cancer. 2012; 12: 436-44.

8. Ramer R, Hinz B. Antitumorigenic targets of cannabinoids - current status and implications. Expert opinion on therapeutic targets. 2016: 1-17.

9. Sarfaraz S, Adhami VM, Syed DN, Afaq F, Mukhtar H. Cannabinoids for cancer treatment: progress and promise. Cancer research. 2008; 68: 339-42.

10.Guzman M. Cannabinoids: potential anticancer agents. Nat Rev Cancer. 2003; 3: 745- 55

11.Galve-Roperh I, Sanchez C, Cortes ML, Gomez del Pulgar T, Izquierdo M, Guzman M. Anti-tumoral action of cannabinoids: involvement of sustained ceramide accumulation and extracellular signal-regulated kinase activation. Nature medicine. 2000; 6: 313-9. 
12. Kampa-Schittenhelm KM, Salitzky O, Akmut F, et al. Dronabinol has preferential antileukemic activity in acute lymphoblastic and myeloid leukemia with lymphoid differentiation patterns. BMC Cancer. 2016; 16: 25.

13. Ligresti A, Moriello AS, Starowicz K, et al. Antitumor activity of plant cannabinoids with emphasis on the effect of cannabidiol on human breast carcinoma. The Journal of pharmacology and experimental therapeutics. 2006; 318: 1375-87.

14.Lombard C, Nagarkatti M, Nagarkatti PS. Targeting cannabinoid receptors to treat leukemia: Role of cross-talk between extrinsic and intrinsic pathways in $\Delta 9$-tetrahydrocannabinol (THC)-induced apoptosis of Jurkat cells. Leukemia Research. 2005; 29: 915-22.

15. Casanova ML, Blazquez C, Martinez-Palacio J, et al. Inhibition of skin tumor growth and angiogenesis in vivo by activation of cannabinoid receptors. The Journal of clinical investigation. 2003; 111: 43-50.

16. Matsuda LA, Lolait SJ, Brownstein MJ, Young AC, Bonner TI. Structure of a cannabinoid receptor and functional expression of the cloned cDNA. Nature. 1990; 346: 561- 4

17. Munro S, Thomas KL, Abu-Shaar M. Molecular characterization of a peripheral receptor for cannabinoids. Nature. 1993; 365: 61-5.

18. Pertwee RG, Howlett AC, Abood ME, et al. International Union of Basic and Clinical Pharmacology. LXXIX. Cannabinoid Receptors and Their Ligands: Beyond CB(1) and CB(2). Pharmacological Reviews. 2010; 62 588-631.

19. Fernandez-Ruiz J, Romero J, Velasco G, Tolon RM, Ramos JA and Guzman M. Cannabinoid CB2 receptor: a new target for controlling neural cell survival? Trends in pharmacological sciences. 2007; 28: 39-45.

20.Atwood BK, Mackie K. CB2: a cannabinoid receptor with an identity crisis. Br J Pharmacol. 2010; 160: 467-79.

21.Golech SA, McCarron RM, Chen Y, et al. Human brain endothelium coexpression and function of vanilloid and endocannabinoid receptors. Brain research Molecular brain research. 2004; 132: 87-92.

22.Ofek O, Karsak M, Leclerc N, et al. Peripheral cannabinoid receptor, CB2, regulates bone mass. Proceedings of the National Academy of Sciences of the United States of America. 2006; 103: 696-701.

23.Zygmunt PM, Petersson J, Andersson DA, et al. Vanilloid receptors on sensory nerves mediate the vasodilator action of anandamide. Nature. 1999; 400: 452-7.

24.Bisogno T, Hanus L, De Petrocellis L, et al. Molecular targets for cannabidiol and its synthetic analogues: effect on vanilloid VR1 receptors and on the cellular uptake and enzymatic hydrolysis of anandamide. BrJ Pharmacol. 2001; 134: 845-52.

25.Hinz B, Ramer R, Eichele K, Weinzierl U, Brune K. Up-regulation of cyclooxygenase-2 expression is involved in $\mathrm{R}(+)$-methanandamideinduced apoptotic death of human neuroglioma cells. Molecular pharmacology. 2004; 66: 1643-51.

26. Ruiz L, Miguel A, Diaz-Laviada I. Delta9-tetrahydrocannabinol induces apoptosis in human prostate PC-3 cells via a receptor-independent mechanism. FEBS Lett. 1999; 458: 400-4

27.Guzman M, Duarte MJ, Blazquez C, et al. A pilot clinical study of Delta9-tetrahydrocannabinol in patients with recurrent glioblastoma multiforme. British journal of cancer. 2006; 95: 197-203.

28. Malfitano AM, Ciaglia E, Gangemi G, Gazzerro P, Laezza C, Bifulco M. Update on the endocannabinoid system as an anticancer target. Expert opinion on therapeutic targets. 2011; 15: 297-308.

29.Joosten M, Valk PJ, Jorda MA, et al. Leukemic predisposition of pSca-1/ Cb2 transgenic mice. Exp Hematol. 2002; 30: 142-9.

30.Nomura DK, Long JZ, Niessen S, Hoover HS, Ng SW, Cravatt BF. Monoacylglycerol lipase regulates a fatty acid network that promotes cancer pathogenesis. Cell. 2010; 140: 49- 61
31. Thors L, Bergh A, Persson E, et al. Fatty acid amide hydrolase in prostate cancer: association with disease severity and outcome, CB1 receptor expression and regulation by IL-4. PLoS One. 2010; 5: e12275.

32.Zheng D, Bode AM, Zhao $\mathrm{Q}$ et al. The cannabinoid receptors are required for ultraviolet-induced inflammation and skin cancer development. Cancer research. 2008; 68: 3992-8.

33. Wang D, Wang H, Ning W, Backlund MG, Dey SK, DuBois RN. Loss of cannabinoid receptor 1 accelerates intestinal tumor growth. Cancer research. 2008; 68: 6468-76.

34. Izzo AA, Aviello G, Petrosino S, et al. Increased endocannabinoid levels reduce the development of precancerous lesions in the mouse colon. Journal of molecular medicine (Berlin, Germany). 2008; 86: 89-98.

35. Salazar M, Carracedo A, Salanueva IJ, et al. Cannabinoid action induces autophagy-mediated cell death through stimulation of ER stress in human glioma cells. The Journal of clinical investigation. 2009; 119: 1359-72.

36. Carracedo A, Lorente M, Egia A, et al. The stress-regulated protein p8 mediates cannabinoid-induced apoptosis of tumor cells. Cancer cell. 2006; 9: 301-12.

37. Galve-Roperh I, Aguado T, Palazuelos J, Guzman M. Mechanisms of control of neuron survival by the endocannabinoid system. Current pharmaceutical design. 2008; 14: 2279-88.

38. Eisenberg-Lerner A, Bialik S, Simon HU, Kimchi A. Life and death partners: apoptosis, autophagy and the cross-talk between them. Cell death and differentiation. 2009; 16: 966-75.

39. Caffarel MM, Sarrio D, Palacios J, Guzman M, Sanchez C. Delta9tetrahydrocannabinol inhibits cell cycle progression in human breast cancer cells through Cdc2 regulation. Cancer research. 2006; 66: 661521.

40. Sanchez C, de Ceballos ML, Gomez del Pulgar T, et al. Inhibition of glioma growth in vivo by selective activation of the $\mathrm{CB}(2)$ cannabinoid receptor. Cancer research. 2001; 61: 5784-9.

41. Gomez del Pulgar T, Velasco G, Sanchez C, Haro A, Guzman M. De novosynthesized ceramide is involved in cannabinoid-induced apoptosis. The Biochemical journal. 2002; 363: 183-8.

42. Encinar JA, Mallo GV, Mizyrycki C, et al. Human p8 is a HMG-I/Y-like protein with DNA binding activity enhanced by phosphorylation. The Journal of biological chemistry. 2001; 276: 2742-51.

43. Du K, Herzig S, Kulkarni RN, Montminy M. TRB3: a tribbles homolog that inhibits Akt/PKB activation by insulin in liver. Science (New York, NY). 2003; 300: 1574-7.

44. Carracedo A, Gironella M, Lorente M, et al. Cannabinoids induce apoptosis of pancreatic tumor cells via endoplasmic reticulum stressrelated genes. Cancer research. 2006; 66: 6748-55.

45. Salazar M, Lorente M, Garcia-Taboada E, et al. The pseudokinase tribbles homologue-3 plays a crucial role in cannabinoid anticancer action. Biochimica et biophysica acta. 2013; 1831: 1573-8.

46. Liu WM, Scott KA, Shamash J, Joel S, Powles TB. Enhancing the in vitro cytotoxic activity of delta9-tetrahydrocannabinol in leukemic cells through a combinatorial approach. Leukemia and Lymphoma. 2008; 49: $1800-9$

47. Powles T, Poele Rt, Shamash J, et al. Cannabis-induced cytotoxicity in leukemic cell lines: the role of the cannabinoid receptors and the MAPK pathway. Blood. 2005; 105: 1214- 21.

48. Greenhough A, Patsos HA, Williams AC, Paraskeva C. The cannabinoid delta(9)-tetrahydrocannabinol inhibits RAS-MAPK and PI3K-AKT survival signalling and induces BAD-mediated apoptosis in colorectal cancer cells. International journal of cancer. 2007; 121: 2172-80.

49. Herrera B, Carracedo A, Diez-Zaera M, Guzmán M, Velasco G. p38 MAPK is involved in CB2 receptor-induced apoptosis of human leukaemia cells. FEBS Letters. 2005; 579: 5084-8. 
50. Blazquez C, Gonzalez-Feria L, Alvarez L, Haro A, Casanova ML, Guzman M. Cannabinoids inhibit the vascular endothelial growth factor pathway in gliomas. Cancer research. 2004; 64: 5617-23.

51. Pandey R, Hegde VL, Nagarkatti M, Nagarkatti PS. Targeting cannabinoid receptors as a novel approach in the treatment of graft-versus-host disease: evidence from an experimental murine model. The Journal of pharmacology and experimental therapeutics. 2011; 338: 819-28.

52. Yeshurun M, Shpilberg O, Herscovici C, et al. Cannabidiol for the Prevention of Graft-versus-Host-Disease after Allogeneic Hematopoietic Cell Transplantation: Results of a Phase II Study. Biology of Blood and Marrow Transplantation. 2015; 21: 1770-5.

53. Singh Y, Bali C. Cannabis Extract Treatment for Terminal Acute Lymphoblastic Leukemia with a Philadelphia Chromosome Mutation. Case Reports in Oncology. 2013; 6: 585-92.
54. McAllister SD, Murase R, Christian RT, et al. Pathways mediating the effects of cannabidiol on the reduction of breast cancer cell proliferation, invasion, and metastasis. Breast cancer research and treatment. 2011; 129: 37-47.

55. McKallip RJ, Lombard C, Fisher M, et al. Targeting CB2 cannabinoid receptors as a novel therapy to treat malignant lymphoblastic disease. Blood. 2002; 100: 627-34

56. Torres S, Lorente M, Rodriguez-Fornes F, et al. A combined preclinical therapy of cannabinoids and temozolomide against glioma. Molecular cancer therapeutics. 2011; 10: 90-103.

57.Donadelli M, Dando I, Zaniboni T, et al. Gemcitabine/cannabinoid combination triggers autophagy in pancreatic cancer cells through a ROS-mediated mechanism. Cell death \& disease. 2011; 2: e152. 